\title{
MODELLING INCONSISTENCY IN THE AHP THROUGH CHOQUET INTEGRATION
}

\author{
Silvia Bortot* and Ricardo Alberto Marques Pereira \\ Dipartimento di Informatica e Studi Aziendali \\ Univers ità di Trento, Via Inama 5, TN 38122 Trento, Italia \\ E-mail: silvia.bortot@,unitn.it; ricalb.marper@unitn.it
}

\begin{abstract}
We propose an extension of Saaty's AHP based on Choquet integration. In our model an appropriate measure of inconsistency is explicitly considered in the aggregation process in order to attenuate (resp. emphasize) the priority values of the criteria with higher (resp. lower) average inconsistency with the remaining criteria.
\end{abstract}

Keywords: AHP, Inconsistency, Capacity, Choquet integral, and Shapley values.

\section{Introduction}

The Analytic Hierarchy Process (AHP), introduced by Thomas L. Saaty (1977, 1986, 1988; Saaty and Vargas, 1991), is a well-known multicriteria aggregation model based on pairwise comparison matrices at two fundamental levels: the lower level encodes pairwise comparison matrices between alternatives (one such matrix for each criterion) and the higher level encodes a single pairwise comparison matrix between criteria. In its most general form, the higher level of the AHP can be structured hierarchically, with several layers of criteria, but in this paper we focus on the single layer case, with a single matrix of pairwise comparis ons between criteria.

The AHP extracts from each pairwise comparison matrix a vector of priority weights corresponding to the principal eigenvector or, alternatively, to the geometric mean vector. In both cases the priority vector has positive components normalized to unit sum. In this paper we consider only the geometric mean method, because its structural properties are more suited for our study. Once the priority vectors associated to the various pairw ise comparison matrices are obtained, the AHP uses the priority vector at the higher level to aggregate (by means of weighted averaging) the lower level priority vectors.

More recently, Saaty proposed the Analytic Network Process (ANP) (1996, 2004a, 2004b; Saaty and Vargas, 2006) in order to incorporate in the AHP the effect of dependence and feedback in the structure of the model, within and between levels. In this paper, instead, we focus on the question of inconsistency and how it can be used to modulate the priority values of the various criteria.

Pairwise comparison matrices are typically inconsistent. In fact, the AHP does not require the decision maker to be consistent, but it might be relevant to estimate his/her degree of inconsistency. Many authors have studied the problem of measuring inconsistency from pairwise comparison matrices. Saaty (1977) proposed a consistency index defined in terms of the principal eighenvalue, Barzilai (1998) proposed the relative error, and in the literature many other indices of consistency have been proposed, see Chu et al.

\footnotetext{
* Corresponding author
} 
(1979), Cavallo and D’Apuzzo (2009, 2010), Peláez and Lamata (2003), Crawford and Williams (1985), Stein and Mizzi (2007), Shiraishi et al. (1998, 2002), Fedrizzi et al. (1990, 2002, 2007).

In order to take into account some form of inconsistency-based interaction between criteria, the Choquet integral (for reviews see Grabisch and Labreuche (2004, 2008, 2010), Grabisch and Kojadinovich (2008)) is an appropriate aggregation operator. The Choquet integral is defined with respect to a (non additive) capacity and generalizes the weighted arithmetic mean (additive case). In order to control the exponential complexity of the model ( $2^{n}-2$ real coefficients are required to define a capacity on a set of $n$ elements), Grabisch (1997a) introduced the so called $k$-additive capacities, see also Grabisch (1997b), and Miranda and Grabisch (1999). The 2-additive case in particular (see Miranda, Grabisch, and Gil, 2005; Mayag, Grabisch, and Labreuche, in press) is a good trade-off between the range of the model and its complexity (only $n(n+1) / 2$ real coefficients are required to define a 2-additive capacity).

In this paper we propose an extension of Saaty's AHP based on Choquet integration with respect to a 2additive capacity: we consider the so-called totally inconsistent matrix induced by Barzilai (1998), and we define a 2-additive capacity on the basis of an appropriate transformation of this matrix. The aggregation scheme is then redefined in terms of the Choquet integration associated to such capacity, thereby extending the usual weighted averaging scheme of Saaty's AHP. A preliminary version of this paper was presented in (Marques Pereira and Bortot, 2004).

An important effect of the new aggregation scheme based on Choquet integration is that of emphasizing (attenuating) the effective priorities of those criteria which have a lower (higher) level of average inconsistency with the remaining ones. This compensatory mechanism that emphasizes some effective priority values and attenuates others is nicely illustrated by the Shapley values associated with the capacity. In our model the Shapley values encode the effective importance weights of the various criteria and, under consistency, the Shapley values coincide with the original priority weights.

\section{Extension of Saaty's AHP}

Consider a finite set of interacting criteria $N=\{1,2, \ldots, n\}$. A capacity is a set function $\mu: 2^{N} \rightarrow[0,1]$ satisfying $\mu(\varnothing)=0, \mu(N)=1$, and the monotonic ity condition: $S \subseteq T \subseteq N \Rightarrow \mu(S) \leq \mu(T)$.

Given a capacity $\mu$, we can define the Choquet integral (Choquet, 1953; Grabisch, 1995, 1996) of a vector $\mathbf{x}=\left(x_{1}, \ldots, x_{n}\right) \in[0,1]^{n}$ with respect to $\mu$ as

$$
\mathrm{C}_{\mu}(\mathbf{x})=\sum_{i=1}^{n}\left[\mu\left(A_{(i)}\right)-\mu\left(A_{(i+1)}\right)\right] x_{(i)}
$$

where the permutation is such that $x_{(1)} \leq x_{(2)} \leq \ldots \leq x_{(n)}$. Moreover $A_{(i)}=\{(i), \ldots,(n)\}$ and $A_{(n+1)}=\varnothing$. Notice that the Choquet integral with respect to an additive capacity $\mu$ reduces to a weighted arithmetic mean, whose weights $w_{i}$ are given by the $\mu(i)$ values. The importance index or Shapley value (Grabisch and Roubens, 1999) of criterion $i \in N$ with respect to $\mu$ is defined as

$$
\phi_{\mu}(i)=\sum_{T \subseteq N \backslash i} \frac{(n-1-t) !(t) !}{n !}[\mu(T \cup i)-\mu(T)], \quad \sum_{i=1}^{n} \phi_{\mu}(i)=1 .
$$

It amounts to a weighted average of the marginal contribution of element $i$ with respect to all coalitions $T \subseteq N \backslash i$ and it can be interpreted as an effective importance weight.

Consider now a positive reciprocal $n \times n$ matrix $\mathbf{A}=\left[a_{i j}\right]$ with $a_{i j}>0$ and $a_{j i}=1 / a_{i j}$ for $i, j=1, \ldots, n$. All pairwise comparison matrices in Saaty's AHP are of this form. However, our model regards only the 
single pairwise comparison matrix between criteria at the higher level of the AHP. This is because that matrix is the one that controls the aggregation process: in Saaty's AHP, the aggregation is performed through a weighted average whose weights are the components of the higher level priority vector.

In general, the positive reciprocal matrix $\mathbf{A}$ above is inconsistent, where consistency means $a_{i j}=a_{i k} a_{k j}$ for all $i, j, k=1, \ldots, n$. However, we can associate to $\mathbf{A}$ a consistent matrix $\tilde{\mathbf{A}}=\left[\tilde{a}_{i j}\right]$ in the following way,

$$
\tilde{a}_{i j}=w_{i} / w_{j} \quad w_{i}=u_{i} / \sum_{j=1}^{n} u_{j} \quad i, j=1, \ldots, n
$$

where $u_{i}$ is the geometric mean of row $i, u_{i}=\sqrt[n]{\prod_{j=1}^{n} a_{i j}}$, and the weights are normalized, $\sum_{i=1}^{n} w_{i}=1$.

Given an element $a_{i j}$ of the matrix $\mathbf{A}$, we define the neighborhood $U\left(a_{i j}\right)$ as the set of elements of row $i$ and column $j$ of the matrix $\mathbf{A}$, that is $U\left(a_{i j}\right)=\left\{a_{i k}, a_{k j} \mid k=1, \ldots, n\right\}$. We say that $a_{i j}$ is locally consistent if, on average, it is cons istent with the elements in its neighborhood,

$$
a_{i j}=\tilde{a}_{i j}=\sqrt[n]{\prod_{k=1}^{n} a_{i k} a_{k j}} \quad i, j=1, \ldots, n .
$$

We now define the scaling function $\mathrm{f}:(0, \infty) \rightarrow(0,1)$ as shown below,

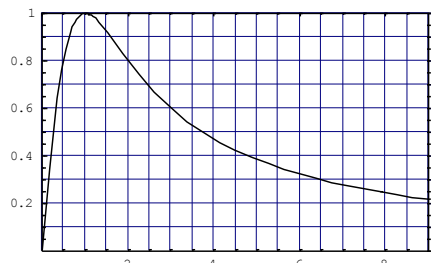

Figure 1. The scaling function $\mathrm{f}(x)=2 /\left(x+x^{-1}\right)$.

Notice that the scaling function $\mathrm{f}$ has a single critical point at $x=1$, where it reaches the maximum value $\mathrm{f}(1)=1$. Moreover, the scaling function $\mathrm{f}$ has the important property $\mathrm{f}(x)=\mathrm{f}\left(x^{-1}\right)$ for all $x>0$.

By means of the scaling function $\mathrm{f}$, we can associate a positive symmetric $n \times n$ matrix $\mathbf{V}=\left[v_{i j}\right]$ to the matrix $\mathbf{A}=\left[a_{i j}\right]$ in the following way,

$$
v_{i j}=\mathrm{f}\left(a_{i j} / \tilde{a}_{i j}\right) \quad v_{i j} \in(0,1] \quad v_{i j}=v_{j i} \quad i, j=1, \ldots, n .
$$

The fact that the $n \times n$ matrix $\mathbf{V}=\left[v_{i j}\right]$ is symmetric is due to the reciprocity of the positive matrix $\mathbf{A}$, plus the fact that $\mathrm{f}(x)=\mathrm{f}\left(x^{-1}\right)$, for $x>0$, since $v_{j i}=\mathrm{f}\left(a_{j i} / \tilde{a}_{j i}\right)=\mathrm{f}\left(\tilde{a}_{i j} / a_{i j}\right)=\mathrm{f}\left(a_{i j} / \tilde{a}_{i j}\right)=v_{i j}$.

Notice that $v_{i j}=1$ if and only if $a_{i j}=\tilde{a}_{i j}$, otherwise $0<v_{i j}<1$ : the more $a_{i j} / \tilde{a}_{i j}$ differs from 1 , the more $v_{i j}$ is close to 0 . Therefore we can consider the matrix $\mathbf{V}=\left[v_{i j}\right]$ as a measure of local consistency.

Moreover, note that our matrix $\mathbf{V}=\left[v_{i j}\right]$ can be regarded as a $[0,1]$-scaled version of the so-called totally inconsistent matrix (Barzilai, 1998) associated with the original pairwise comparison matrix $\mathbf{A}=\left[a_{i j}\right]$.

Given a general (typically inconsistent) positive reciprocal matrix $\mathbf{A}=\left[a_{i j}\right]$, one can define a 2-additive capacity $\mu: 2^{N} \rightarrow[0,1]$ in the following way: making use of the Möbius transform $m_{\mu}$ of the capacity $\mu$, we define $m_{\mu}(i)=2 w_{i} /(v+1)$ for each singlet $\{i\}$ and $m_{\mu}(i j)=-2 w_{i}\left(1-v_{i j}\right) w_{j} /(v+1)$ for each doublet $\{i, j\}$, with null higher order terms, where $v_{i}=\sum_{j=1}^{n} v_{i j} w_{j}$ and $v=\sum_{i=1}^{n} v_{i} w_{i}$ denote weighted averages of local consistency values, with $w_{i}<v_{i} \leq 1$ for $i=1, \ldots, n$ and $\sum_{i=1}^{n} w_{i}^{2}<v \leq 1$. Then, we define the value of the 2-additive capacity $\mu$ on a coalition $S$ as the sum of the singletons and doublets contained in the coalition $S$, as given by the Möbius transform $m_{\mu}$,

$$
\mu(S)=\sum_{\{i\} \subseteq S} 2 w_{i} /(v+1)+\sum_{\{i, j\} \subseteq S}\left(-2 w_{i}\left(1-v_{i j}\right) w_{j}\right) /(v+1)
$$


In particular, we have

$$
\mu(i)=2 w_{i} /(v+1) \quad \mu(i j)=\left(2 w_{i}+2 w_{j}-2 w_{i}\left(1-v_{i j}\right) w_{j}\right) /(v+1) \quad i, j=1, \ldots, n .
$$

The capacity $\mu$ satisfies the boundary conditions $\mu(\emptyset)=0, \mu(N)=1$, and is monotonic and subadditive. The (strict) monotonicity of the capacity is guaranteed by the fact that the positive value $w_{i}$ associated to each node of the graph dominates (in absolute value) the sum of the negative values $-w_{i}\left(1-v_{i j}\right) w_{j}$ associated to the $n-1$ edges connecting that node with the other nodes in the graph,

$$
w_{i}-\sum_{j=1}^{n} w_{i}\left(1-v_{i j}\right) w_{j}=w_{i}-w_{i}\left(1-v_{i}\right)=w_{i} v_{i}>w_{i}^{2}>0 \quad i=1, \ldots, n .
$$

This model is an extension of Saaty's AHP: if the matrix $\mathbf{A}$ is consistent, then the capacity $\mu$ is additive and the Choquet integral coincides with a weighted arithmetic mean whose weights are as in Saaty's AHP. Using the Möbius transform, one can easily compute the Shapley values $\phi_{i}, i=1, \ldots, n$ associated with the capacity $\mu$ defined above,

$$
\phi_{i}=m_{\mu}(i)+\frac{1}{2} \sum_{j \in N \backslash i} m_{\mu}(i j)=\frac{2}{v+1}\left(w_{i}-\frac{1}{2} \sum_{j \in N \backslash i} w_{i}\left(1-v_{i j}\right) w_{j}\right)=w_{i} \frac{1+v_{i}}{v+1} \quad i=1, \ldots, n .
$$

In our multicriteria aggregation model the Shapley values encode the effective importance weights of the various criteria. When the matrix $\mathbf{A}$ is consistent, we have $v_{i j}=1$ for all $i, j=1, \ldots, n$ and the equation above implies that the Shapley values are $\phi_{i}=w_{i}$. Otherwise, when $\mathbf{A}$ is inconsistent, we have $\phi_{i}>w_{i}$ if $v_{i}>v$ and $\phi_{i}<w_{i}$ if $v_{i}<v$. In general, the fact that $\mathbf{A}$ is inconsistent changes the original distribution of weights, attenuating the importance values of the more inconsistent criteria (those with higher average inconsistency) and emphasizing the importance values of the more consistent criteria.

In fact, if we compute the second order Taylor expansion of the Shapley values $\phi_{i}=w_{i}\left(1+v_{i}\right) /(1+v)$, $i=1, \ldots, n$, around the consistency condition $v_{i} \approx 1$, we get

$$
\phi_{i} \approx w_{i}\left(1+\frac{1}{4}\left(v_{i}-v\right)(3-v)\right) \quad i=1, \ldots, n .
$$

Notice that the second order approximation of the Shapley values is still normalized to unit sum, since $\sum_{i=1}^{n} w_{i}\left(v_{i}-v\right)=0$. Moreover, the Taylor expansion shows clearly that, in the small inconsistency approximation, we have $\phi_{i}>w_{i}$ if $v_{i}>v$ and $\phi_{i}<w_{i}$ if $v_{i}<v$, in a compensatory mechanism typical of weighted averaging schemes.

\section{REFERENCES}

Barzilai, J. (1998). Consistency measures for pairwise comparison matrices. Journal of Multi-Criteria Decision Analysis, 7(3), 123-132.

Cavallo, B., \& D'Apuzzo, L. (2009). A general unified framework for pairwise comparison matrices in multicriterial methods. International Journal of Intelligent Systems, 24(4), 377-398.

Cavallo, B., \& D'Apuzzo, L. (2010). Characterizations of cons istent pairwise comparison matrices over abelian linearly ordered groups. International Journal of Intelligent Systems, 25(10), 1035-1059.

Choquet, G. (1953). Theory of capacities. Annales de l'Institut Fourier, 5, 131-295. 
Chu, A.T.W., Kalaba, R.E., \& Springarn, K. (1979). A comparison of two methods for determining the weights of belonging to fuzzy sets. Journal of Optimization Theory and Applications, 27(4), 531-538.

Crawford, G., \& Williams, C. (1985). A note on the analys is of subjective judgment matrices. Journal of Mathematical Psychology, 29(4), 387-405.

Fedrizzi, M. (1990). On a consensus measure in a group MCDM problem. In: J. Kacprzyk, M. Fedrizzi (Eds), Multiperson Decision Making Models using Fuzzy Sets and Possibility Theory, Kluwer Academic Publishers 231-241.

Fedrizzi, M., Fedrizzi, M., \& Marques Pereira, R.A. (2002). On the issue of consistency in dynamical consensual aggregation. In: B. Bouchon Meunier, J. Gutierrez Rios, L. Magdalena, R. R. Yager (Eds), Technologies for Constructing Intelligent Systems, Physica-Verlag (Springer) 129-137.

Fedrizzi, M., \& Giove, S. (2007). Incomplete pairwise comparisons and consistency optimization. European Journal of Operational Research, 183(1), 303-313.

Grabisch, M. (1995). Fuzzy integral in multicriteria decision making. Fuzzy Sets \& Systems, 69, 279-298.

Grabisch, M. (1996). The application of fuzzy integrals in multicriteria decision making. European Journal of Operational Research, 89(3), 445-456.

Grabisch, M. (1997a). $k$-order additive discrete fuzzy measures and their representation. Fuzzy Sets and Systems, 92(2), 167-189.

Grabisch, M. (1997b). Alternative representations of discrete fuzzy measures for decision making. International Journal of Uncertainty, Fuzziness and Knowledge-Based Systems, 5(5), 587-607.

Grabisch, M., Kojadinovich, I., \& Meyer, P. (2008). A review of methods for capacity identification in Choquet integral based multi-attribute utility theory: Applications of the Kappalab R package. European Journal of Operational Research, 186(2), 766-785.

Grabisch, M., \& Labreuche, C. (2004). Fuzzy measures and integrals in MCDA. In: J. Figue ira, S. Greco and M. Ehrgott (Eds), Multiple Criteria Decision Analysis, Kluwer Academic Publishers 563-608.

Grabisch, M, \& Labreuche, C. (2008). A decade of application of the Choquet and Sugeno integrals in multi-criteria decision aid. $4 O R, 6(1), 1-44$.

Grabisch, M., \& Labreuche, C. (2010). A decade of application of the Choquet and Sugeno integrals in multi-criteria decision aid. Annals of Operations Research, 175(1), 247-286.

Grabisch, M., \& Roubens, M. (1999). An axiomatic approach to the concept of interaction among players in cooperative games. International Journal of Game Theory, 28(4), 547-565.

Marques Pereira, R.A., \& Bortot, S. (2001). Consensual Dynamics, Stochastic Matrices, Choquet Measures, and Shapley Aggregation. Proc. 22nd Linz Seminar on Fuzzy Set Theory: Valued Relations and Capacities in Decision Theory, Linz, Austria, 78-80.

Marques Pereira, R.A., \& Bortot, S. (2004). Choquet measures, Shapley values, and inconsistent pairwise comparison matrices: an extension of Saaty's A.H.P.. Proc. 25th Linz Seminar on Fuzzy Set Theory: Mathematics of Fuzzy Systems, Linz, Austria, 130-135. 
Mayag, B., Grabisch, M., \& Labreuche, C. A representation of preferences by the Choquet integral with respect to a 2-additive capacity. Theory and Decision, (in press).

Mayag, B., Grabisch, M., \& Labreuche, C. A characterization of the 2-additive Choquet integral through cardinal information. Fuzzy Sets and Systems, (in press).

Miranda, P., \& Grabisch, M. (1999). Optimization issues for fuzzy measures. International Journal of Uncertainty, Fuzziness and Knowledge-Based Systems, 7(6), 545-560.

Miranda, P., Grabisch, M., \& Gil, P. (2005). Axiomatic structure of $k$-additive capacities. Mathematical Social Sciences, 49(2), 153-178.

Peláez, J.I., \& Lamata, M.T. (2003). A new measure of inconsistency for positive reciprocal matrices. Computers and Mathematics with applications, 46(12), 1839-1845.

Saaty, T.L. (1977). A scaling method for priorities in hierarchical structures. Journal of Mathematical Psychology, 15, 234-281.

Saaty, T.L. (1986). Axiomatic foundation of the analytic hierarchy process. Management Science, 32(7), 841-855.

Saaty, T.L. (1988). Multicriteria Decision Making: The Analytic Hierarchy Process. RWS Publications, Pittsburgh PA, Original version published by McGraw-Hill, 1980.

Saaty, T.L. (1996). Decision Making with Dependence and Feedback: The Analytic Network Process. RWS Publications, Pittsburgh, PA.

Saaty, T.L. (2004a). Decision Making - the Analytic Hierarchy Process and the Analytic Network Process (AHP/ANP). Journal of Systems Science and Systems Engineering, 13(1), 1-35.

Saaty, T.L. (2004b). Fundamentals of the Analytic Network Process - dependence and feedback in decision-making with a single network. J. of Systems Science and Systems Engineering, 13 (2), 129-157.

Saaty, T.L., \& Vargas, L.G. (1991). Prediction, Projection and Forecasting. Kluwer Academic Publishers, Norwell, MA.

Saaty, T.L., \& Vargas, L.G. (2006). Decision Making with the Analytic Network Process: Economic, Political, Social and Technological Applications with benefits, Opportunities, Costs and Risks. Springer's International Series in Operations Research \& Management Science.

Shiraishi, S., Obata, T., \& Daigo, M. (1998). Properties of a positive reciprocal matrix and their application to AHP. Journal of the Operations Research Society of Japan, 41 (3), 404-414.

Shiraishi, S., \& Obata, T. (2002). On a maximization problem arising from a positive reciprocal matrix in the AHP. Bulletin of Informatics and Cybernetics, 34(2), 91-96.

Stein, W.E., \& Mizzi, P.J. (2007). The harmonic consistency index for the analytic hierarchy process. European Journal of Operational Research, 177(1), 488-497. 